\title{
Identification of QTLs affecting scopolin and scopoletin biosynthesis in Arabidopsis thaliana
}

\author{
Joanna Siwinska', Leszek Kadzinski', Rafal Banasiuk', Anna Gwizdek-Wisniewska', Alexandre Olry 2,3, \\ Bogdan Banecki ${ }^{1}$, Ewa Lojkowska ${ }^{1}$ and Anna Ihnatowicz ${ }^{1 *}$
}

\begin{abstract}
Background: Scopoletin and its glucoside scopolin are important secondary metabolites synthesized in plants as a defense mechanism against various environmental stresses. They belong to coumarins, a class of phytochemicals with significant biological activities that is widely used in medical application and cosmetics industry. Although numerous studies showed that a variety of coumarins occurs naturally in several plant species, the details of coumarins biosynthesis and its regulation is not well understood. It was shown previously that coumarins (predominantly scopolin and scopoletin) occur in Arabidopsis thaliana (Arabidopsis) roots, but until now nothing is known about natural variation of their accumulation in this model plant. Therefore, the genetic architecture of coumarins biosynthesis in Arabidopsis has not been studied before.
\end{abstract}

Results: Here, the variation in scopolin and scopoletin content was assessed by comparing seven Arabidopsis accessions. Subsequently, a quantitative trait locus (QTL) mapping was performed with an Advanced Intercross Recombinant Inbred Lines (Al-RILs) mapping population EstC (Est- $1 \times$ Col). In order to reveal the genetic basis of both scopolin and scopoletin biosynthesis, two sets of methanol extracts were made from Arabidopsis roots and one set was additionally subjected to enzymatic hydrolysis prior to quantification done by high-performance liquid chromatography (HPLC). We identified one QTL for scopolin and five QTLs for scopoletin accumulation. The identified QTLs explained 13.86\% and 37.60\% of the observed phenotypic variation in scopolin and scopoletin content, respectively. In silico analysis of genes located in the associated QTL intervals identified a number of possible candidate genes involved in coumarins biosynthesis.

Conclusions: Together, our results demonstrate for the first time that Arabidopsis is an excellent model for studying the genetic and molecular basis of natural variation in coumarins biosynthesis in plants. It additionally provides a basis for fine mapping and cloning of the genes involved in scopolin and scopoletin biosynthesis. Importantly, we have identified new loci for this biosynthetic process.

Keywords: Coumarins, Natural variation, Plant-environment interaction, Scopoletin, Scopolin, Secondary metabolism, QTL mapping

\section{Background}

Plants produce a great variety of secondary metabolites. It is estimated that between 4000 to 20000 metabolites per species can be expected [1]. This great biochemical diversity reflects the variety of environments in which plants live, and the way they have to deal with different environmental stimuli. The production of specialized secondary metabolites is assumed to protect plants against biotic and abiotic stresses [2]. Although Arabidopsis is a small plant with

\footnotetext{
* Correspondence: anna.ihnatowicz@biotech.ug.edu.pl

${ }^{1}$ Intercollegiate Faculty of Biotechnology of University of Gdansk and Medical University of Gdansk, ul. Kladki 24, Gdansk 80-822, Poland

Full list of author information is available at the end of the article
}

short generation time and highly reduced genome, it has a set of secondary metabolites that is as abundant and diverse as those of other plant taxa [3]. In recent years, this model plant was extensively used towards identification of genes and enzymes working in a complex network involved in secondary metabolites biosynthesis and regulation [4].

Currently, genetic variation found between natural Arabidopsis accessions is an important basic resource for plant biology [5-7]. Arabidopsis with its extensive genetic natural variation provides an excellent model to study variation in the biosynthesis of secondary metabolites in natural populations. Recent genetic analysis of natural 
variation in untargeted metabolic composition uncovered many qualitative and quantitative differences in metabolite accumulation between Arabidopsis accessions [8-10]. Numerous studies [8,10-12] proved the presence of abundant genetically controlled variation for various classes of secondary metabolites. Coumarins (scopoletin, scopolin, skimmin and esculetin) are one of the secondary metabolite classes found in Arabidopsis' roots [13-16]. But up to now, nothing is known about natural variation in coumarins content between Arabidopsis accessions.

Coumarins are a group of important natural compounds that provide for the plant antimicrobial and antioxidative activities, and are produced as a defence mechanism against pathogen attack and abiotic stresses [17]. Importantly, coumarins are widely recognized in the pharmaceutical industry for their wide range of therapeutic activities and are an active source for drug development. Numerous coumarins have medical application in the treatment of burns and rheumatoid diseases. Furanocoumarins, which are coumarin derivatives, are used in the treatment of leucoderma, vitiligo and psoriasis [18], due to their photoreactive properties. Moreover, they are used in symptomatic treatment of demyelinating diseases, particularly multiple sclerosis [19]. Furanocoumarin-producing plants that are currently studied are non-model organisms [20] and many approaches to identify the genes underlying genetic variation in coumarins accumulation are not yet available in those species. Scopoletin, which is a major coumarin compound of Arabidopsis, has been found in many plant species [21-29], and was clearly shown to have antifungal and antibacterial activities important for medical purposes [30]. All these properties make coumarins attractive from the commercial point of view.

Coumarins are derived from phenylopropanoid pathway, which serves as a rich source of metabolites in plants [31,32]. It was suggested that in Arabidopsis several branch pathways leading from phenylpropanoid compounds to coumarins are probable [14]. Scopoletin and scopolin biosynthesis was shown to be strongly dependent on the CYP98A3 [14], which is the cytochrome P450 catalyzing 3 '-hydroxylation of p-coumarate units in the phenylpropanoid pathway [33]. The feruloyl-CoA was suggested to be a major precursor in scopoletin biosynthesis [15]. A key enzyme involved in the final step of scopoletin biosynthesis, which is the conversion of feruloyl-CoA into 2-hydroxyferuloyl-CoA, is encoded by a member of the iron (Fe) IIand 2-oxoglutarate-dependent dioxygenase (2OGD) family, designated as F6' $\mathrm{H} 1$ [15]. Despite the advances that have been made in previous years [15,34-42] (Figure 1), many questions with regard to coumarins biosynthesis are still open [43]. In particular, the regulation of the biosynthesis of coumarins is not well understood. Up to now, all studies investigating coumarins biosynthesis in the model plant Arabidopsis were done with one laboratory accession
Col-0, which was used as the genetic background of all mutant and transgenic plants.

To gain an understanding of the genetic architecture of coumarins biosynthesis, we screened a set of Arabidopsis accessions for variation in scopolin and scopoletin content, and subsequently conducted a quantitative trait locus (QTL) mapping. Our study addressed the following questions. Is there a natural variation in accumulation of scopolin and scopoletin between Arabidopsis accessions and what are genetic regions responsible for the observed differences? What are candidate genes possibly underlying QTLs involved in scopolin and scopoletin biosynthesis?

\section{Results}

\section{Phenotypic variation between accessions}

A set of seven natural Arabidopsis accessions, which are the parents of existing RIL populations and represent accessions from different locations, were used in the initial screening for variation in scopolin and scopoletin accumulation. Accessions were grown in vitro in liquid cultures in order to obtain the optimal growth of plant roots. Under these conditions, most of the scopoletin is stored in root cells in vacuoles as its glycoside form, scopolin. In order to reveal the content of both scopolin and that of scopoletin, a subset of the methanol extracts made from Arabidopsis roots were subjected to enzymatic hydrolysis in order to hydrolyze the glycoside forms of coumarins. Using highperformance liquid chromatography (HPLC), we detected in the roots scopoletin (sct in Figure 2), as well as scopolin (scl in Figure 2BC). The identification of scopoletin in HPLC fraction (Figure 3A) was further confirmed using gas chromatography/mass spectrometry (GC/MS) by comparison to spectrum library (Figure $3 \mathrm{~B}$ ). The quantification of coumarins in methanol root extracts made from seven Arabidopsis accessions clearly showed the presence of natural variation in scopolin content before enzymatic hydrolysis (Figure 4A) and scopoletin after hydrolysis (Figure 4B). In spite of the fact that scopolin standard was not available and in order to unify further analysis, we measured the amounts of both scopolin and scopoletin as area\% of total chromatogram signals. The statistically significant differences between group means for scopolin and scopoletin accumulation were determined by one-way ANOVA ( $p<0.001$ and $\mathrm{p}<0.0001$, respectively). Values that are not significantly different based on the post hoc test (least significant differences [LSD]) are indicated by the same letters (Figure 4). Based on the obtained results we have selected an Advanced Intercross Recombinant Inbred Lines (AI-RILs) mapping population derived from the cross between Col-0 and Est-1, because these parents significantly differed in coumarins content. Further genetic analysis was performed using values for the accumulation of scopolin before enzymatic hydrolysis and the content of scopoletin after hydrolysis of methanol extracts. 


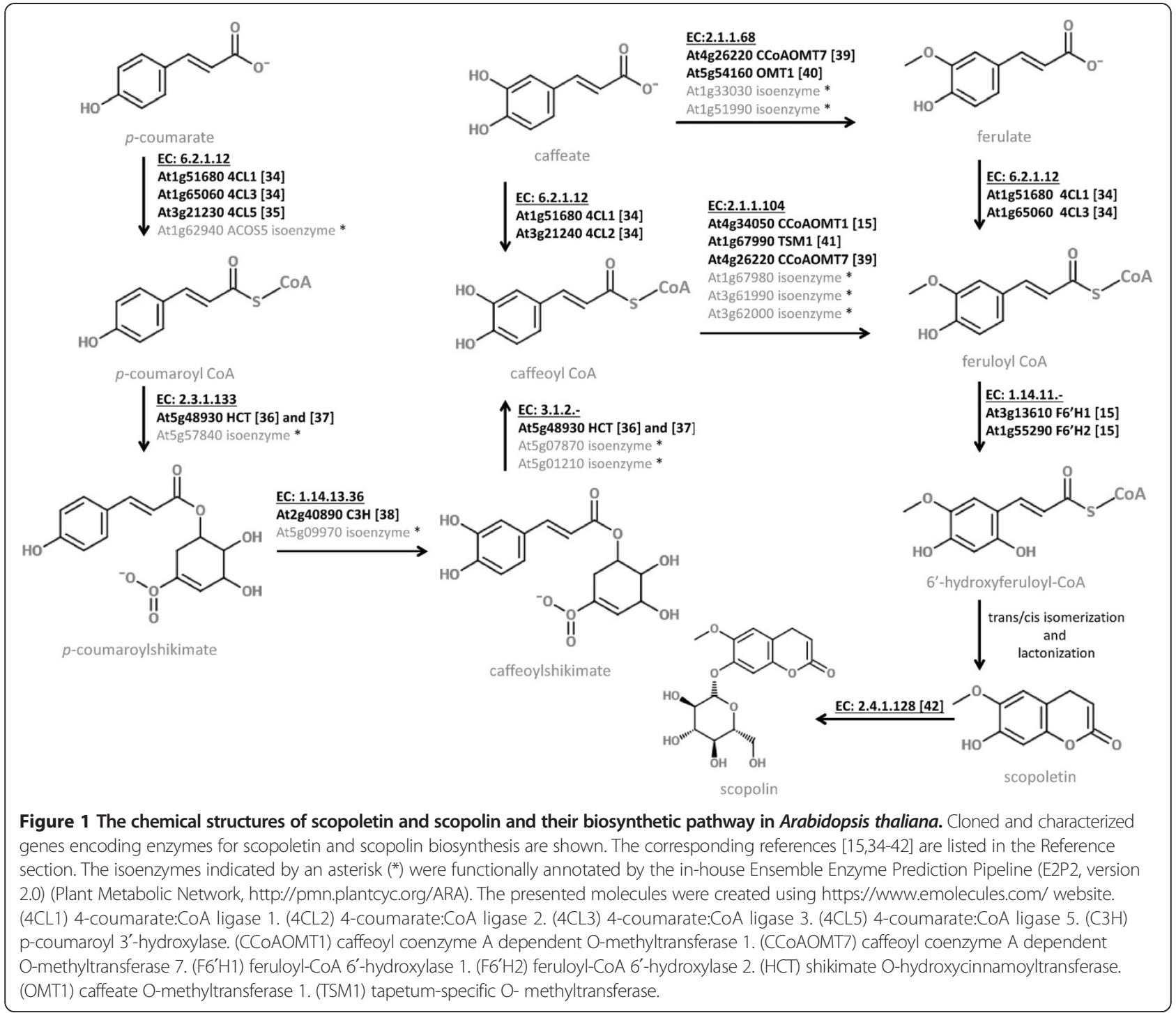

Genetic analyses of scopolin and scopoletin accumulation The scopoletin and scopolin content values were determined for three biological replicates of AI-RILs ( $n=144$ and $n=140$, respectively) and parental lines, which were grown in independent flasks in liquid cultures. A set of lines (AI-RILs) showed a wider range of scopolin (Figure 5A) and scopoletin (Figure 5B) values than the ones observed for both parental lines (Col-0 and Est-1), which indicated the presence of transgressive segregation and suggested that multiple loci contribute to variation in the EstC population. The lowest scopolin content within AI-RILs was 1.90 (measured as an area\% of total chromatogram signals) that corresponds to $20 \%$ of the minimum Col-0 value. The maximal relative value of scopolin was 45.13 , which corresponds to $159 \%$ of the maximal Est- 1 value. For scopoletin content, these values were respectively 7.82 (54\% of the minimum Col-0 value) and 54.93 (159\% of the maximal Est-1 value) (Table 1). Having a commercially available scopoletin standard, we were able to quantify the scopoletin contents as $\mu \mathrm{g} / \mathrm{g}$ fresh weight $(\mu \mathrm{g} / \mathrm{gFW})$ in both parental lines of the AI-RILs mapping population (Col-0 and Est-1) before and after enzymatic hydrolysis. The scopoletin levels in root samples not subjected to hydrolysis were $\sim 3 \mu \mathrm{g} / \mathrm{gFW}$ and $\sim 10 \mu \mathrm{g} / \mathrm{gFW}$ in Col-0 and Est-1 respectively, and $\sim 16 \mu \mathrm{g} / \mathrm{gFW}$ and $\sim 86 \mu \mathrm{g} / \mathrm{gFW}$ in samples after hydrolysis. These values correspond to $\sim 18,54$, 82 and $449 \mathrm{nmol} / \mathrm{gFW}$ respectively that is in the range found in the literature data, which vary from $\sim 1$ to $1200 \mathrm{nmol} / \mathrm{gFW}$ depending on plant culture being used [14]. The calculated quantities of parental lines (Table 2) can be used as references for the overall quantity of the products in the whole mapping population.

In order to identify the fraction of variation that is genetically determined, the broad sense heritability $\left(H^{2}\right)$ for scopolin and scopoletin content was estimated as described in Methods section. In the AI-RIL population, 

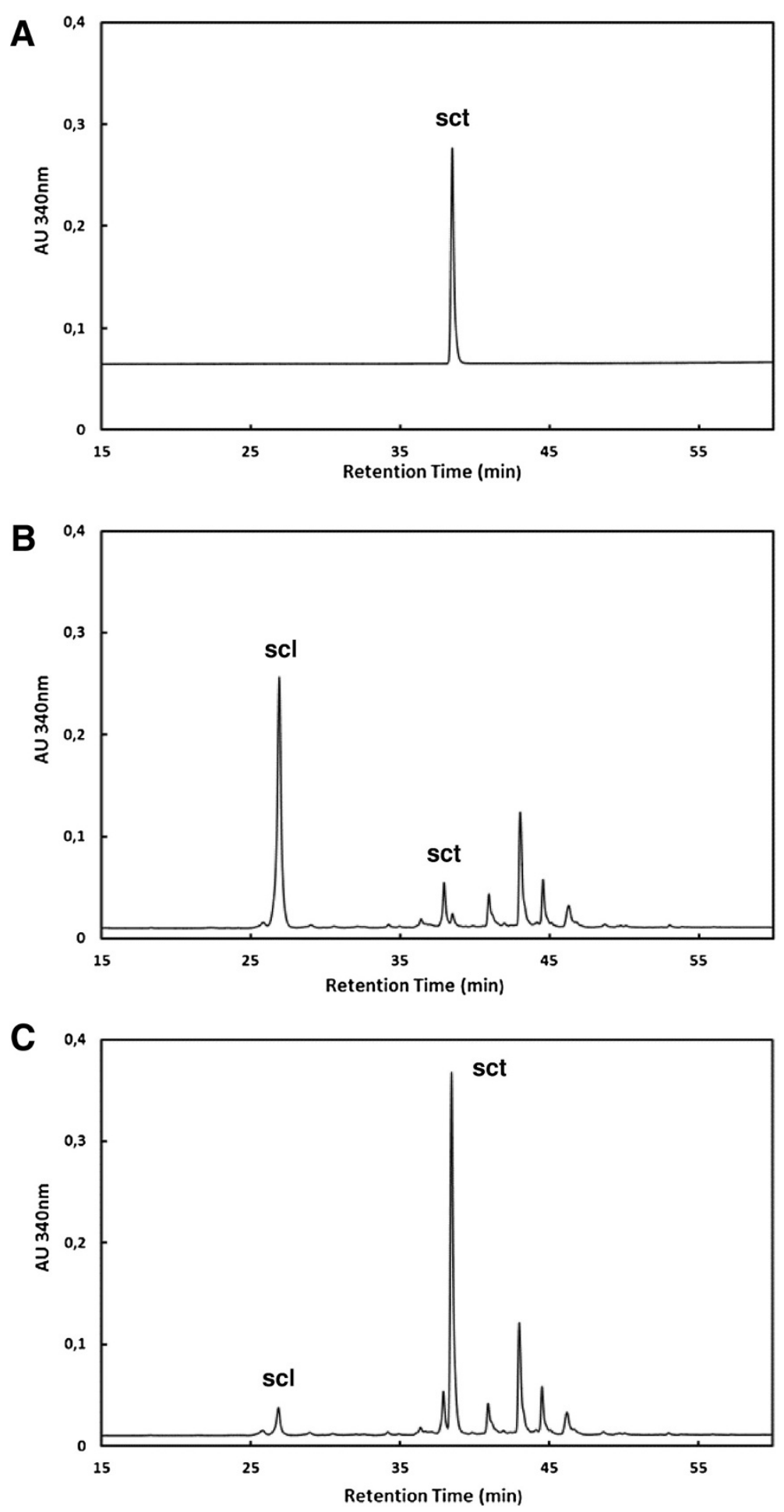

Figure 2 Chromatograms of scopoletin standard and methanol extracts from Arabidopsis thaliana roots. The column effluent was monitored with fluorescence detector with excitation at $340 \mathrm{~nm}$ and emission at $460 \mathrm{~nm}$. The peak for glucoside of scopoletin - scopolin (scl); the peak for scopoletin (sct). (A) Chromatogram of scopoletin standard. (B) Chromatogram of methanol extract from Arabidopsis roots before enzymatic hydrolysis. (C) Chromatogram of methanol root extract subjected to hydrolysis using $\beta$-glucosidase. The peak for scopoletin is a dominant peak of total chromatogram. 


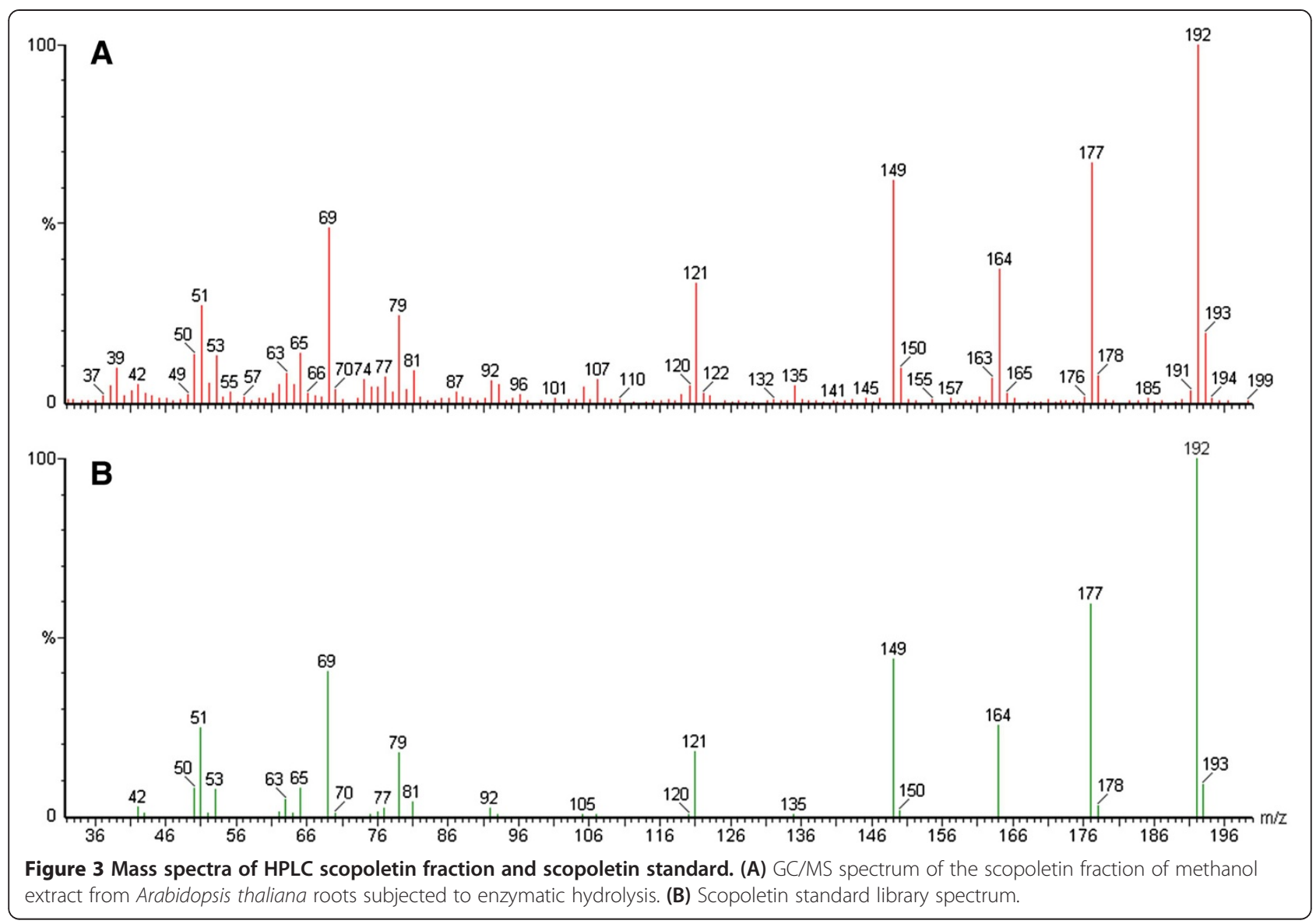

the broad sense heritability ranged from 0.45 for scopoletin to 0.50 for scopolin content (Table 1 ). To explore the relationship between scopolin content in methanol root extracts before enzymatic hydrolysis and scopoletin levels in extracts subjected to hydrolysis, the mean values of coumarins for each AI-RILs were used as phenotype values in trait correlation analysis. A relatively strong genetic correlation $\left(R^{2}=0.6634\right)$ was observed between the level of coumarins measured before and after hydrolysis in the AI-RILs population, indicating genetic co-regulation of scopolin and scopoletin biosynthesis (Figure 6).

\section{Mapping QTLs for scopolin and scopoletin accumulation}

Six QTLs were identified, with one QTL being detected for scopolin and five QTLs for scopoletin accumulation (Table 3). The QTL effect sizes ranged from the $7.0 \%$ to $16.7 \%$ of the phenotypic variance explained by the QTL (PVE), with three of the six QTLs having effect sizes below 10\% PVE. One QTL (SCL1) was detected for scopolin accumulation at the bottom of chromosome 5 (Figure 7) explaining the 13.86\% PVE (Table 3), and five QTLs (SCT1 - SCT5) for scopoletin accumulation were identified on chromosome 1, 3 and 5 (Figure 8, Table 3). No QTLs were detected on chromosome 2 and 4 . To improve the QTL model explaining variation in a scopoletin content, the MQM approach was performed using two QTLs (SCT4 and SCT5) as cofactors. We have included in the model QTL on chromosome 1 (SCT1), despite its LOD score was slightly below the threshold (3.327). The whole model explains $37.6 \%$ variance for scopoletin content. No epistasis between the main effect loci were detected.

\section{QTL mapping identifies known and new loci for coumarins biosynthesis}

Some of the mapped QTLs underlying variation in scopolin (SCL1) and scopoletin (SCT1 and SCT2) accumulation in the AI-RILs population, co-localize with the genes annotated to be involved in coumarin biosynthetic process (Plant Metabolic Network, http://plantcyc.org/, Figure 1). We detected seven cloned and characterized genes encoding enzymes for scopoletin and scopolin biosynthesis that co-localize with detected QTLs (see Additional file 1). Within the SCL1 interval, which is characterized by one of the highest LOD score values, there are two very good candidates. One of them is At5g48930 encoding a shikimate O-hydroxycinnamoyltransferase (HCT), while the other one (At5g54160) encodes caffeic acid/5-hydroxyferulic acid O-methyltransferase (OMT1). Importantly, both genes are 


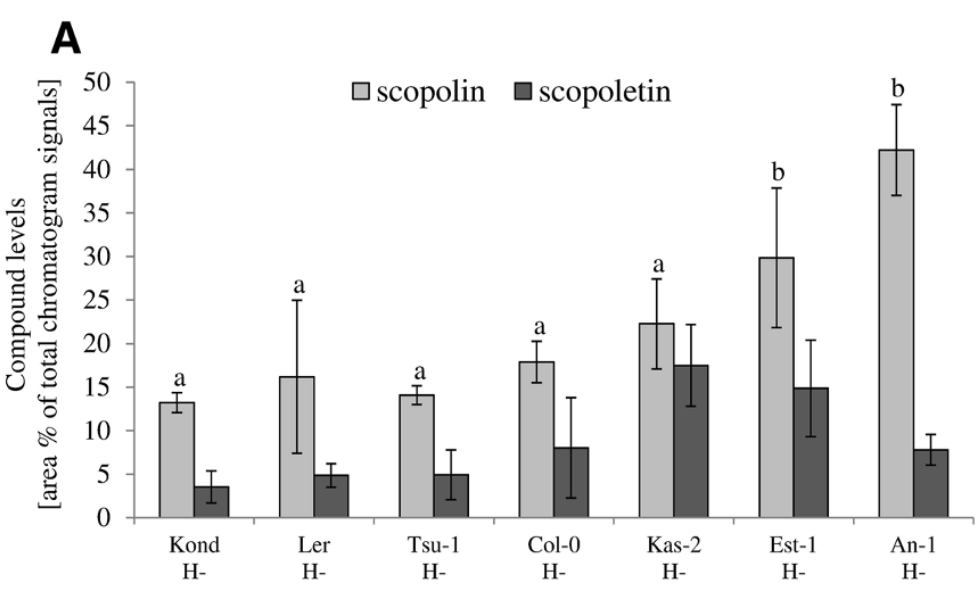

\section{B}

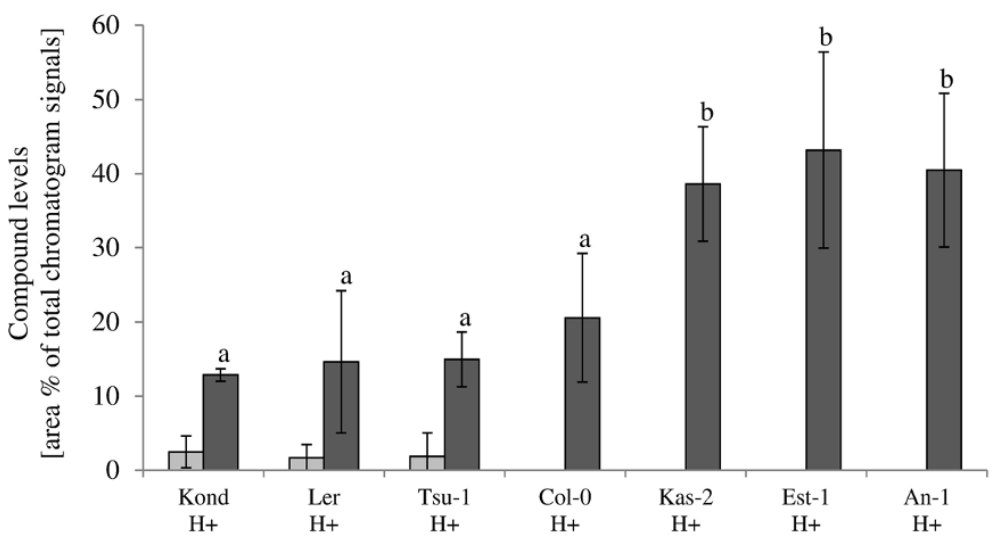

Arabidopsis thaliana accessions

Figure 4 Relative levels of scopolin and scopoletin in the roots of seven Arabidopsis thaliana accessions. (A) Scopolin level in methanol root extracts without hydrolysis $(\mathrm{H}-$ ). (B) Scopoletin content in the methanol extracts that were subjected to enzymatic hydrolysis $(\mathrm{H}+)$ prior to quantification. The statistically significant differences between group means for scopolin and scopoletin accumulation were determined by one-way ANOVA ( $p<0.001$ and $p<0.0001$, respectively). Values that are not significantly different based on the post hoc test (least significant differences [LSD]) are indicated by the same letters. The data analysis consisted of scopolin and scopoletin relative levels measured as area\% of total chromatogram signals. Error bars represent the SD from three measurements.

expressed in roots (SCL1 in Table 4). Within the SCT1 and SCT2 intervals underlying variation in scopoletin content more possible candidate genes were detected: At1g33030, At1g51990, At1g67980 and At1g67990 (TSM1) encoding proteins from O-methyltransferase family; At1g51680 and At1g65060 encoding isoforms of 4-coumarate:CoA ligase (4CL1 and 4CL3 respectively); At1g62940 encoding acylCoA synthetase (ACOS5); and At1g55290 encoding feruloyl CoA ortho-hydroxylase 2 ( $\left.6^{\prime} \mathrm{H} 2\right)$.

In order to reveal other candidate genes possibly underlying detected QTLs, two QTLs for scopoletin content (SCT4 and SCT5) and one QTL associated with scopolin (SCL1) accumulation were chosen for further in silico analyses. The selected intervals are characterized by the highest percentage of phenotypic variance explained by each QTL and the highest LOD score values. The annotated functions for all genes located in the selected QTL intervals were checked. As a result, we selected genes encoding transcription factors that might be induced by environmental stresses and enzymes that according to the annotation functions could be possibly involved in scopolin and scopoletin biosythensis. Subsequently, we performed in silico analysis of the tissue distribution and level of expression of selected genes. Only genes that were expressed in roots were selected as possible candidates for further studies. As a result, we selected a set of genes that deserve close attention as possible new loci underlying variation in scopolin and scopoletin accumulation (Table 4). Among candidates possibly involved in scopoletin accumulation, a particularly interesting one is a CYP81D11 gene (At3g28740) encoding a member of the cytochrome P450 family, which is located within the QTL on chromosome 3 (SCT4 in 

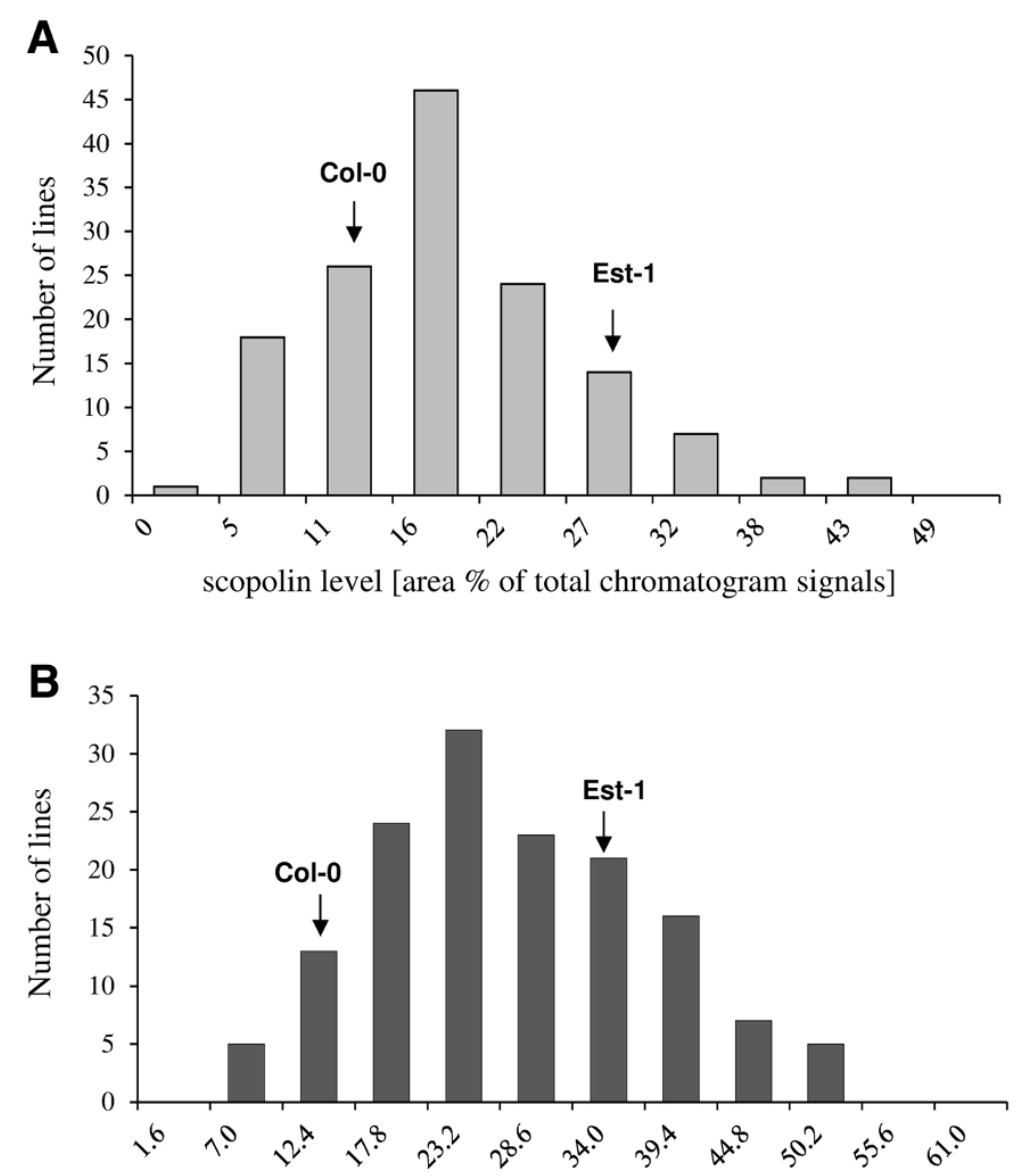

scopoletin level [area \% of total chromatogram signals]

Figure 5 Frequency distribution of scopolin and scopoletin relative levels in the Al-RILs and parental lines roots. Plants used for genetic mapping were grown in in vitro liquid cultures under a photoperiod of $16 \mathrm{~h} \mathrm{light}\left(35 \mu \mathrm{mol} \mathrm{m} \mathrm{m}^{-2} \mathrm{~s}^{-1}\right)$ at $20^{\circ} \mathrm{C}$ and $8 \mathrm{~h}$ dark at $18^{\circ} \mathrm{C}$. Coumarins content in the roots of the Al-RILs population and parental lines (accessions Col-0 and Est-1) were determined by HPLC. (A) Scopolin contents were determined in methanol extracts without hydrolysis. (B) Methanol extracts subjected to enzymatic hydrolysis were used for scopoletin quantification. The data analysis consisted of scopolin and scopoletin relative levels measured as area\% of total chromatogram signals. The average values of Col-0 and Est- 1 are indicated with arrows.

Table 4). According to the 1001 Genomes Project database (www.1001genomes.org) and re-sequencing data of Est-1 from our laboratory (see Additional files 2 and 3, indicated as Est-1*), the CYP81D11 gene contains several SNPs and one indel in the coding sequences of the parental lines of EstC mapping population and in the other accessions tested in this study (see Additional file 2).
Other interesting candidates are three genes (At5g14340, At5g14750, At5g15130) located within the QTL interval on chromosome 5 (SCT5 in Table 4), which encode members of the MYB and WRKY transcription factor families. These genes are relatively highly expressed in roots and their expression is induced by various environmental stresses [44]. A particularly interesting candidate that

Table 1 Parental values, ranges and heritabilities in the AI-RILs of scopolin and scopoletin content (relative values ${ }^{\mathrm{a}}$ )

\begin{tabular}{|c|c|c|c|c|c|}
\hline \multirow[b]{2}{*}{ Trait } & \multicolumn{2}{|l|}{ Parents } & \multicolumn{3}{|l|}{ AI-RIL } \\
\hline & Col-0 value ${ }^{a}$ & Est-1 value $^{a}$ & Range & Mean & Heritability $^{\mathrm{d}}$ \\
\hline Scopolin (H-) & 9.71 & 28.45 & $1.9-45.13$ & 19.84 & 0.50 \\
\hline Scopoletin $(\mathrm{H}+)^{c}$ & 14.58 & 34.53 & $7.82-54.93$ & 29.68 & 0.45 \\
\hline
\end{tabular}

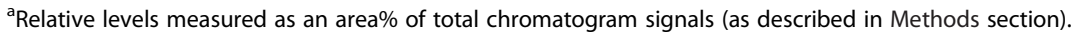

${ }^{\mathrm{b} C o n t e n t}$ of scopolin before enzymatic hydrolysis.

${ }^{\mathrm{C}}$ Content of scopoletin after enzymatic hydrolysis.

${ }^{\mathrm{d}}$ Measure of total phenotypic variance attributable to genetic differences among genotypes (broad sense heritability) calculated as $V_{G} /\left(V_{G}+V_{E}\right)$. 
Table 2 The quantified levels of scopoletin ${ }^{a}$ in Arabidopsis thaliana roots

\begin{tabular}{|c|c|c|c|c|}
\hline & \multicolumn{2}{|c|}{ Roots $(\mu \mathrm{g} / \mathrm{gFW})$} & \multicolumn{2}{|c|}{ Roots (nmol/gFW) } \\
\hline & Col-0 value ${ }^{a}$ & Est-1 value ${ }^{\mathrm{a}}$ & Col-0 value $^{a}$ & Est-1 value $^{a}$ \\
\hline Scopoletin $(\mathrm{H}-)^{\mathbf{b}}$ & $3.4 \pm 1.8$ & $10.4 \pm 2.4$ & $17.7 \pm 9.4$ & $54.1 \pm 6.2$ \\
\hline Scopoletin $\left(\mathrm{H}_{+}\right)^{\mathrm{c}}$ & $15.8 \pm 6.4$ & $86.2 \pm 9.8$ & $82.2 \pm 33.3$ & $448.6 \pm 51.0$ \\
\hline
\end{tabular}

${ }^{\mathrm{a}}$ Scopoletin was quantified with HPLC. Values show the averages of scopoletin contents with standard deviation (SD) from two measurements.

${ }^{\mathrm{b} C}$ Content of scopoletin before enzymatic hydrolysis.

${ }^{c}$ Content of scopoletin after enzymatic hydrolysis.

could be possibly linked to scopolin accumulation was detected within the QTL on chromosome 5 (SCL1 in Table 4). It is At5g53990 encoding a UDP-glycosyltransferase, which is relatively highly expressed in Arabidopsis roots [44]. According to the 1001 Genomes Project and our resequencing data of Est-1, this gene contains several SNPs in the coding sequences of tested accessions including the parental lines (see Additional file 3). Interestingly, the CYP81D11 and UDP-glycosyltransferase sequences originating from Est, Est-1 (both taken from the 1001 Genomes Project database) and Est-1* that was re-sequenced in our laboratory are not identical (see Additional files 2 and 3). This needs to be further verified.

\section{Discussion}

Here, we report a QTL mapping study of variation in scopoletin and scopolin accumulation between two Arabidopsis accessions and thereby we demonstrate the

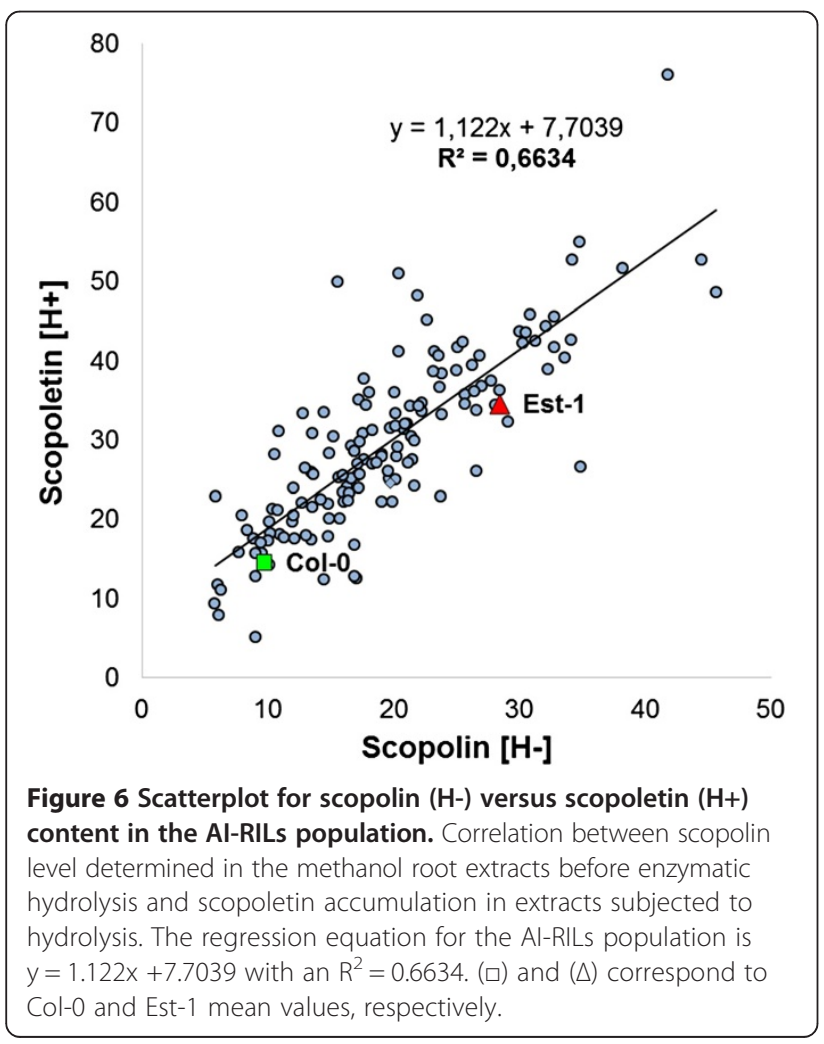

usefulness of Arabidopsis natural variation in elucidating the genetic and molecular basis of coumarins biosynthesis.

A large number of Arabidopsis recombinant inbred line (RIL) populations are available and extensively used for identification of numerous QTLs controlling various traits such as growth, development or resistance to different biotic and abiotic stresses as well as the content of chemical compounds $[5,7,9,45,46]$. In most studies, the average number of QTLs identified is between one and 10 and at least one major QTL is detected [47]. Here, one QTL for scopolin and five QTLs for scopoletin accumulation were detected, which is in agreement with the average result in the field. Using an AI-RILs mapping population has the advantage in comparison to RILs due to the fact that the opportunity for recombination is increased before genotypes are fixed upon selfing [48]. As a result, using AI-RILs mapping population that captures an increased number of recombination events [48], enabled us to detect QTLs with effect size as low as 7.0\% PVE.

Once QTL has been identified, the next challenge is to identify the gene(s) underlying detected QTL. In most cases, a large number of genes that are present in the QTL interval cannot be directly tested for candidacy. In order to reduce the mapped region, a fine-mapping is performed in which many individuals are genotyped for markers around the QTL. More accurate QTL localization might lead to the selection of candidate genes. Nonetheless, performing a fine mapping may be practically difficult if the QTL effect is relatively small [49]. When multiple data sets are available, which is the case for Arabidopsis, it is possible to improve accuracy and to test the candidacy of genes within mapped QTL intervals [49] based on the available information. Therefore, it seems like a realistic possibility to identify candidate genes underlying a QTL by using the high throughput expression data and the complete genome sequences of numerous Arabidopsis accessions that were used to construct mapping populations. There are successful examples of using expression arrays in identifying genes causally associated with quantitative traits of interest, both in plants and animals [50,51]. In this study, possible candidate genes were found within mapped QTL intervals for scopolin and scopoletin content, including known and novel loci. Further functional analysis, including re-sequencing, characterization of loss- 
Table 3 Characteristics of the detected QTLs underlying scopolin and scopoletin biosynthesis in AI-RILs population

\begin{tabular}{llllllll}
\hline Trait & QTL & Chr $^{\mathbf{a}}$ & LOD score & Peak $^{\mathbf{b}}(\mathbf{c M})$ & Confidence interval $^{\mathbf{c}}$ (cM) & Confidence interval (bp) $^{\text {PVE }^{\mathbf{d}}(\%)}$ \\
\hline Scopolin & SCL1 & 5 & 4.53 & 174.2 & $173.6-185.9$ & $19.414 .594-22.027 .830$ & 13.86 \\
Scopoletin & SCT1 & 1 & 3.327 & 71.1 & $32.6-178.6$ & $4.826 .763-20.083 .545$ & 7.008 \\
& SCT2 & 1 & 3.594 & 189.3 & $176.5-263.2$ & $19.672 .910-28.537 .561$ & 7.602 \\
& SCT3 & 3 & 4.223 & 19.2 & $6.7-25.8$ & $786.303-4.140 .699$ & 9.027 \\
& SCT4 & 3 & 7.427 & 96.7 & $93.8-99.0$ & $9.942 .057-10.995 .480$ & 16.735 \\
& SCT5 & 5 & 5.249 & 53.3 & $51.7-53.9$ & $4.235 .132-5.725 .918$ & 11.409 \\
\hline
\end{tabular}

${ }^{\mathrm{a} C h r o m o s o m e ~ n u m b e r ~}$

${ }^{\mathrm{b}}$ Position of peak.

${ }^{c} 1$-LOD support interval.

${ }^{d}$ Percentage of phenotypic variance explained by the QTL (PVE).

of-function alleles and conducting gene complementation either by crossing or genetic transformation, are required to prove the role of selected possible candidate genes in coumarins biosynthesis and their regulation.

Expanding molecular understanding of coumarins biosynthesis at an ecological level will be beneficial for the future discovery of the physiological mechanisms of action of genes involved in coumarins biosynthesis. It was suggested recently that some members the 2'-OG dioxygenase family, including the F6' $\mathrm{H} 1$ that is a key enzyme in scopoletin biosynthesis, may be involved in Fe deficiency responses and metabolic adjustments linked to Fe homeostasis in plant cells [52]. Other latest studies showed that Fe deficiency induces the secretion of scopoletin and its derivatives by Arabidopsis roots [53], and that F6' $\mathrm{H} 1$ is required for the biosynthesis of coumarins that are released into the rhizosphere as part of the strategy I-type Fe acquisition machinery [54]. Previously, the existence of natural variation in root exudation profiles was clearly detected among eight Arabidopsis accessions [55]. The

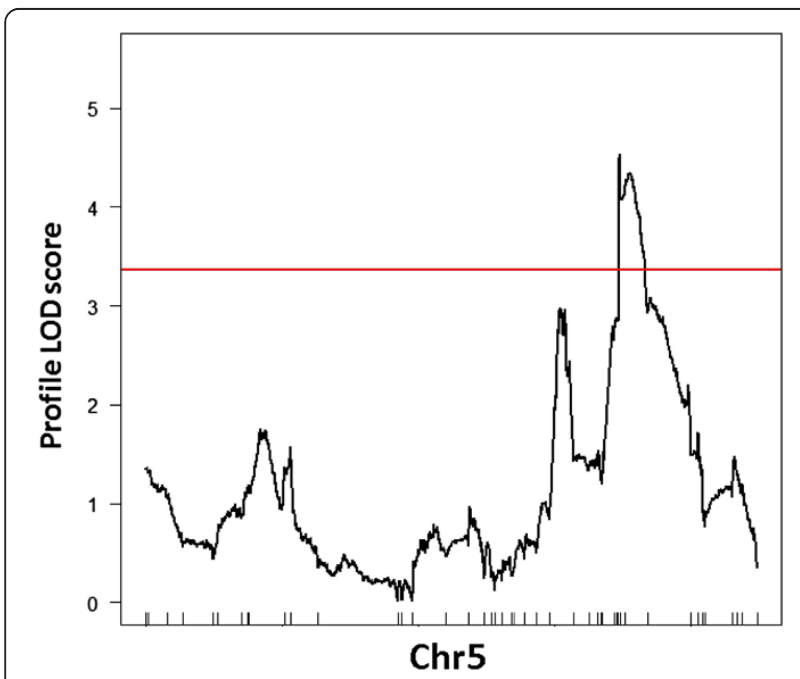

Figure 7 LOD profile for QTL underlying scopolin accumulation in the Al-RILs. One-dimensional LOD profile for the QTL underlying variation in scopolin accumulation (SCL1). Red line represents LOD threshold (3.4). above mentioned findings make a study of coumarins biosynthesis in Arabidopsis using naturally occurring intraspecific variation even more promising and up-to-date.

\section{Conclusions}

In summary, we have presented here for the first time a presence of naturally occurring intraspecies variation in scopoletin and its glucoside, scopolin, accumulation among seven Arabidopsis accessions. Even though, these accessions do not completely represent a wide genetic variation existing in Arabidopsis, it is assumed that these accessions should reflect genetic adaptation to local environmental factors [6]. A QTL mapping study of scopoletin and scopolin variation within EstC mapping population was conducted leading to the identification of new loci. The results presented here suggest that natural variation in coumarins content in Arabidopsis has a complex molecular basis. Importantly, they also provide a basis for fine mapping and cloning of the genes involved in coumarins biosynthesis.

\section{Methods}

\section{Plant material}

Seven Arabidopsis thaliana accessions Antwerpen (An-1, Belgium), Columbia (Col-0, Germany), Estland (Est-1, Estonia), Kashmir (Kas-2, India), Kondara (Kond, Tadjikistan), Landsberg erecta (Ler, Poland) and Tsu (Tsu-1, Japan), which are the parents of existing RIL populations and represent accessions from different locations, were used in the initial screening for variation in scopoletin and scopolin accumulation. An advanced recombinant inbred lines (AI-RILs) mapping population (EstC) derived from the cross between Columbia (Col-0) and Estland (Est-1) was used in the QTL mapping experiment [48]. All seeds of the Arabidopsis accessions and mapping population were kindly provided by Maarten Koornneef from the Max Planck Institute for Plant Breeding Research in Cologne, Germany. Arabidopsis accessions are available at the stock centre NASC (http://arabidopsis.info/). The EstC mapping population together with the marker data are available at the NASC under the stock number CS39389. 


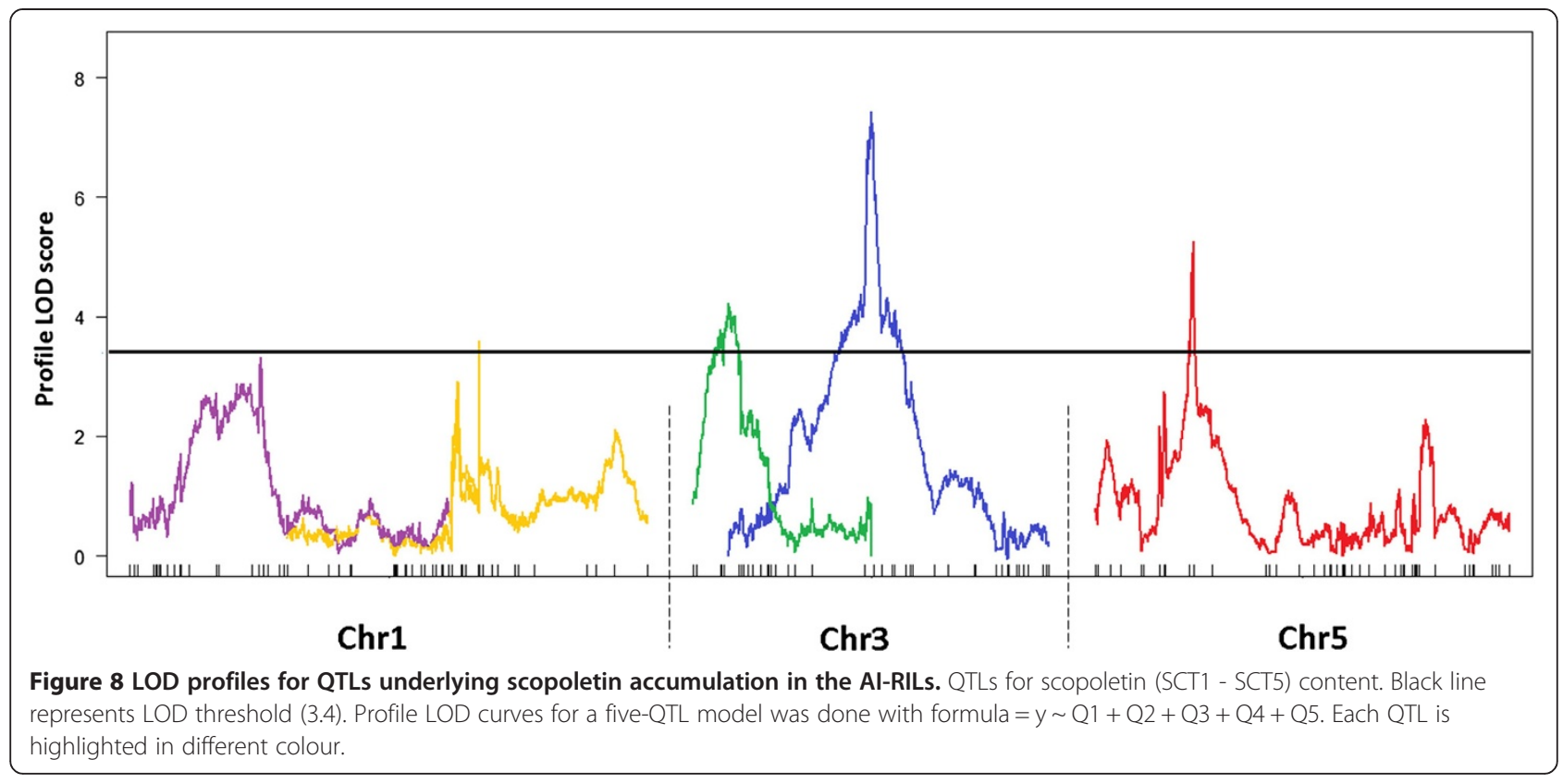

\section{Growth conditions}

The seeds were surface sterilized by soaking in $70 \%$ ethanol for two min and subsequently kept in 5\% calcium hypochlorite solution for eight min. Afterwards seeds were rinsed three times in autoclaved millipore water and planted on 0.5 Murashige and Skoog's (MS) medium containing $1 \%$ sucrose, $0.8 \%$ agar supplemented with $100 \mathrm{mg} / \mathrm{l}$ myo-inositol, $1 \mathrm{mg} / \mathrm{l}$ thiamine hydrochloride, $0.5 \mathrm{mg} / \mathrm{l}$ pyridoxine hydrochloride and $0.5 \mathrm{mg} / \mathrm{l}$ nicotinic acid. For stratification, plates were kept in the dark at $4^{\circ} \mathrm{C}$ for $72 \mathrm{~h}$ and then placed under defined growth conditions. All plants were grown in vitro in plant growth chambers under a photoperiod of $16 \mathrm{~h}$ light $\left(35 \mu \mathrm{mol} \mathrm{m}{ }^{-2} \mathrm{~s}^{-1}\right)$ at $20^{\circ} \mathrm{C}$ and $8 \mathrm{~h}$ dark at $18^{\circ} \mathrm{C}$. After 10 days seedlings were transferred from agar plates into $200 \mathrm{ml}$ glass culture vessels $(5.5 \mathrm{~cm}$ diameter $\times 10 \mathrm{~cm}$ high, glass jars with magenta B caps) containing $8 \mathrm{ml}$ sterile liquid medium. Plants grown in liquid cultures were incubated on rotary platform shakers at $120 \mathrm{rpm}$. After 17 days plants were harvested $\left(28^{\text {th }}\right.$ day of culture), leaves and roots were frozen separately in liquid nitrogen and stored at $-80^{\circ} \mathrm{C}$. All genotypes were grown in three biological replicates (in independent flasks). The growth conditions were monitored by a HOBO U12 data logger (Onset Computer Corporation, Bourne, MA) that recorded the parameters (temperature, light intensity and relative humidity) in an interval at every five minutes.

\section{Preparation of methanol extracts from Arabidopsis roots}

The root tissue was homogenized using steel beads and sonication. The coumarins were extracted at $4^{\circ} \mathrm{C}$ with $80 \%$ methanol. After $24 \mathrm{~h}$ two sets of methanol extracts were centrifuged for $20 \mathrm{~min}$ at $13000 \mathrm{rpm}$, one set was additionally subjected to enzymatic hydrolysis using $\beta$ - glucosidase from almonds (Sigma-Aldrich) dissolved in acetate buffer according to modified protocol of [56].

\section{Scopoletin and scopolin quantification by High- Performance Liquid Chromatography (HPLC)}

The methanol extracts of Arabidopsis roots with and without enzymatic treatment were analyzed (Figure 2) using a Perkin Elmer series 200 HPLC system comprising of a quaternary LC pump, autosampler, column oven and a UV detector. All samples were filtered with $0.22 \mu \mathrm{m}$ filters before loading. The volume injected was $10 \mu \mathrm{l}$. Gradient elution on Perkin Elmer C18 column SC18 $(250 \times 4.6 \mathrm{~mm})$ was performed at flow rate of $0.7 \mathrm{ml} / \mathrm{min}$ with the following solvent system: (A) $50 \mathrm{mM}$ ammonium acetate $\mathrm{pH} 4.5$, (B) Methanol: starting from 30\% B for 2 min, 30-80\% B in $40 \mathrm{~min}$ followed by isocratic elution and column regeneration. The fluorescence detector was based on absorbance at $340 \mathrm{~nm}$ excitation wavelength and emission at $460 \mathrm{~nm}$. The data analysis consisted of scopoletin and scopolin relative analysis (area percent of total chromatogram).

\section{Scopoletin identification by Gas Chromatography/Mass Spectrometry (GC/MS)}

The HPLC fractions containing scopoletin peak were collected and scopoletin identification was confirmed (Figure 3A) with Gas Chromatography/Mass Spectrometry (GC/MS) by comparison to spectrum library (Figure 3B). GC/MS analysis was performed using a Perkin-Elmer GC XL Gas Chromatograph interfaced to a Mass Spectrometer equipped with an Elite-5MS (5\% diphenyl/ 95\% dimethyl polysiloxane $)$ fused to a capillary column $(30 \times 0.25 \mu \mathrm{m}$ ID $\times 0.25 \mu \mathrm{m} \mathrm{df}$ ). For GC/MS detection, an electron ionization system operated in electron impact mode 
Table 4 Potential candidate genes ${ }^{\mathrm{a}}$ located within the SCL1, SCT4 and SCT5 intervals No. Locus: Description (TAIR)

Gene expression level (Arabidopsis eFP

Browser)

Relative level (Fold-change) Absolute level

Candidate genes selected from the QTL interval (SCT4) on chromosome 3: 9942057 to 10995480 nt.

1. AT3G27230: S-adenosyl-L-methionine-dependent methyltransferases superfamily protein 1.18

210.15

2. AT3G27325: Hydrolases, acting on ester bond

3. AT3G27340: Molecular_function unknown; involved in oxidation reduction

4. AT3G27890: Encodes NAD(P)H:quinone reductase 367.30

5. AT3G28200: Peroxidase superfamily protein 242.03

6. AT3G28480: Oxoglutarate/iron-dependent oxygenase 130.66

7. AT3G28740: Encodes a member of the cytochrome p450 family (CYP81D11) 35.84

Candidate genes selected from the QTL interval (SCT5) on chromosome 5: 4235132 to $5725918 \mathrm{nt}$.

8. AT5G13780: Acyl-CoA N-acyltransferases (NAT) superfamily protein 1.96

395.68

9. AT5G14130: Peroxidase superfamily protein

10. AT5G14240: Thioredoxin superfamily protein

11. AT5G14340: Member of the R2R3 factor gene famil (MYB40)

12. AT5G14390: Alpha/beta-Hydrolases superfamily protein

1.36

69.43

13. AT5G14430: S-adenosyl-L-methionine-dependent methyltransferases superfamily protein

14. AT5G14750: Encodes a MyB-related protein containing R2 and R3 repeats (MYB66)

15. AT5G15130: Encodes a member of WRKY Transcription Factor (WRKY72)

16. AT5G15180: Peroxidase superfamily protein

Candidate genes selected from the QTL interval (SCL1) on chromosome 5: 19414594 to $22027829 \mathrm{nt}$.

17. AT5G47950: HXXXD-type acyl-transferase family protein 11.16

$68^{*}$

18. AT5G47980: HXXXD-type acyl-transferase family protein

19. AT5G47990: Encodes a member of the CYP705A family of cytochrome P450 enzymes

40.50

$168^{*}$

20. AT5G48000: Encodes a member of the CYP708A family of cytochrome P450 enzymes

21. AT5G48020: 2-oxoglutarate (2OG) and Fe(II)-dependent oxygenase superfamily protein

22. AT5G48560: Basic helix-loop-helix (bHLH) DNA-binding superfamily protein

23. AT5G48930: Encode shikimate O-hydroxycinnamoyltransferase $(H C T)^{\text {b }}$

24. AT5G49520: Encodes WRKY48, a member of the WRKY Transcription Factor

25. AT5G49560: Putative methyltransferase family protein

26. AT5G49810: Methionine S-methyltransferase (MMT)

27. AT5G49950: Alpha/beta-Hydrolases superfamily protein

28. AT5G50890: Alpha/beta-Hydrolases superfamily protein

29. AT5G51130: S-adenosyl-L-methionine-dependent methyltransferases superfamily protein

30. AT5G51880: 2-oxoglutarate (2OG) and Fe(II)-dependent oxygenase superfamily protein

1.41

46.65

0.99

291.46

31. AT5G52260: Encodes a member of the R2R3 factor gene family (MYB19)

$30.21^{*}$

32. AT5G52400: Encodes a member of CYP715A

33. AT5G53560: Encodes a cytochrome b5 isoform that can be reduced by AtCBR

34. AT5G53990: UDP-Glycosyltransferase superfamily protein

$10.75^{\#}$

35. AT5G54080: Homogentisate 1,2-dioxygenase (HGO)

${ }^{a}$ The list of potential candidate genes was compiled by searching TAIR (http://www.arabidopsis.org/) and Arabisopsis eFP Browser (http://bar.utoronto.ca/).

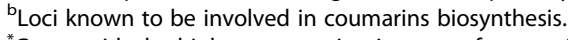

"Genes with the highest expression in roots of vegetative rosette.

\#Genes with relatively high expression in roots of vegetative rosette.

The selected intervals are associated with scopolin (SCL1) and scopoletin (SCT4, SCT5) accumulation and are characterized by the highest percentage of phenotypic variance explained by each QTL and the highest LOD score values. Most of selected genes (except two highlighted with letter ${ }^{b}$ ) are novel loci. 
with an ionization energy of $70 \mathrm{eV}$. Helium gas was used as a carrier gas at a constant flow rate of $1 \mathrm{ml} / \mathrm{min}$, and an injection volume of $2 \mu \mathrm{l}$ was employed (a split ratio of $10: 1)$. The ion-source temperature was $250^{\circ} \mathrm{C}$, the oven temperature was programmed from $100^{\circ} \mathrm{C}$ (isothermal for $5 \mathrm{~min}$ ), with an increase of $10^{\circ} \mathrm{C} / \mathrm{min}$ to $300^{\circ} \mathrm{C}$. Mass spectra were taken at $70 \mathrm{eV}$; a scan interval of $0.5 \mathrm{~s}$ and fragments from 30 to $450 \mathrm{Da}$. The solvent delay was 1 to $2 \mathrm{~min}$, and the total GC/MS running time was $38 \mathrm{~min}$. The mass-detector used in this analysis was Turbo-Mass GoldPerkin-Elmer, and the MS software Turbo-Mass ver-5.1.

\section{Quantitative traits}

Coumarins were quantified in the methanol root extracts of three biological replicates (cultivated in independent flasks) of all AI-RILs individuals. Methanol extracts subjected to enzymatic hydrolysis were used for scopoletin quantification, while scopolin contents were determined in methanol extracts without hydrolysis.

\section{Quantitative genetic analyses}

The scopolin and scopoletin mean values for each AIRILs were used in QTL mapping and trait correlation analysis. The regression equation and $R^{2}$ were calculated by plotting scopolin and scopoletin mean values against one another in Scatterplot (Microsoft Excel). The broad sense heritability $\left(H^{2}\right)$ was estimated according to the formula $H^{2}=V_{G} /\left(V_{G}+V_{E}\right)$, where $V_{G}$ is the amonggenotype variance component and $V_{E}$ is the residual (error) variance.

\section{QTL analyses in the AI-RIL population}

Statistical analysis of phenotypic data was performed by Shapiro-Wilk normality test. Phenotypic data is normally distributed at the significance level $\alpha=0.05$. QTL mapping was performed using $\mathrm{R}$ software (A Core Team, 2012, www.R-project.org) with R/qtl package [57,58]; http://www. rqtl.org/). QTL mapping was performed with Simple Interval Mapping (SIM) (data not shown) followed by the Multiple QTL mapping (MQM) procedure. The QTLs with the highest logarithm of odds (LOD) scores detected by SIM were subsequently used to make the QTL model by the MQM. The final QTL model was done with the backward elimination of cofactors with the window size $10 \mathrm{cM}$ and maximum number of cofactors 5. Significance threshold (LOD) values $(\mathrm{P}<0.05)$ for the QTL presence was estimated from 10000 permutations and is 3.4. "Addint" function has been used to add pairwise interaction, one at a time, to a multiple-QTL model. No interaction has been detected.

\section{Candidate genes selection}

The physical positions of genes annotated to be involved in coumarin biosynthetic process (Plant Metabolic Network, http://plantcyc.org/) were checked according to TAIR (http://www.arabidopsis.org/). To reveal other candidate genes possibly underlying detected QTLs, a list of candidates was constructed using the following criteria: (1) genes encoding enzymes belonging to families involved in coumarins biosynthesis and genes encoding transcription factors that might be induced by environmental stresses (http://www.arabidopsis.org/); (2) genes that are expressed in roots (http://bar.utoronto.ca/). The list of potential candidates was compiled by searching TAIR (http://www. arabidopsis.org/) and Arabisopsis eFP Browser (http://bar. utoronto.ca/) (Table 4).

\section{Statistical analysis}

All treatments included at least three (or two in case of parental lines used in the genetic mapping) biological replicates. Data processing and statistical analyses (one way ANOVA, post-hoc test: least significant difference test [LSD]) were carried out using Microsoft Excel. Error bars representing standard deviation (SD) are shown in the figures; the data presented are means.

\section{DNA samples preparation and sequencing}

The RNeasy ${ }^{\circ}$ Plant Mini Kit (Qiagen) was used following the instructions of the manufacturer and including on-column DNA digestion step with the RNase-Free DNase Set (Qiagen) to eliminate genomic DNA contamination. $0.5 \mu \mathrm{g}$ of RNA was used for reverse transcription by Maxima First Strand cDNA Synthesis Kit (Thermo Scientific). The amplification of genes coding sequences was carried out in a $20 \mu \mathrm{l}$ reaction mixture containing cDNA synthetized from RNA isolated from roots, $0.4 \mathrm{U}$ of Platinum ${ }^{\circ}$ Taq DNA Polymerase (Invitrogen), $200 \mu \mathrm{M}$ $\mathrm{dNTP}, 1 \mu \mathrm{M}$ primers, and $1 \times \mathrm{PCR}$ Buffer and $1.5 \mathrm{mM} \mathrm{Mg}^{2+}$. The reaction mixture was denatured at $94^{\circ} \mathrm{C}$ for $2 \mathrm{~min}$, and then the PCR amplification was performed using 34 cycles of $94^{\circ} \mathrm{C}$ for $30 \mathrm{sec}, 52^{\circ} \mathrm{C}$ for $30 \mathrm{sec}$, and $72^{\circ} \mathrm{C}$ for $90 \mathrm{sec}$ in the Thermal Cycler C1000 Touch (Bio-Rad). Gene-specific primers used for AT5G53990 UDPglycosyltransferase amplification were 5' - ATGGGCCAA AATTTTCACGCT -3' and 5' - TCATTCAAGATTTGTA TCGTTGACT-3' and for AT3G28740 CYP81D11 5'ATGTCATCAACAAAGACAATAATGG-3' and 5' - TTA TGGACAAGAAGCATCTAAAACC-3'. PCR products were cloned into pCR8 vector (Invitrogen). For plasmid amplification and maintenance, the Escherichia coli strain One Shot $^{\oplus}$ (Invitrogen) was used. Positive clones were sequenced using vector specific primers M13fwd and M13rev and BigDye Terminator v3.1 (Life Technologies). Sequencing reaction products were separated and analyzed by 3730xl DNA Analyzer. All sequences were aligned using CLUSTALW [59]. 


\section{Availability of supporting data}

The data sets supporting the results of this article are included within the article and its additional files.

\section{Additional files}

Additional file 1: Figure S1. The position of known loci involved in scopolin and scopoletin biosynthesis.

Additional file 2: Figure S2. Multiple Sequence Alignment of coding sequences of AtCYP81D11 gene produced by CLUSTALW.

Additional file 3: Figure S3. Multiple Sequence Alignment of coding sequences of AtUDP-glycosyltransferase gene produced by CLUSTALW.

\begin{abstract}
Abbreviations
2OGD: 2-oxoglutarate-dependent dioxygenase; 4CL1: 4-coumarate:CoA ligase 1; 4CL2: 4-coumarate:CoA ligase 2; 4CL3: 4-coumarate:CoA ligase 3; 4CL5: 4-coumarate:CoA ligase 5; ACOS5: Acyl-CoA synthetase 5; Al-RILs: Advanced intercross recombinant inbred lines; $\mathrm{C} 3 \mathrm{H}$ : $\mathrm{p}$-coumaroyl 3'-hydroxylase; CCOAOMT1: Caffeoyl coenzyme A dependent O-methyltransferase 1; CCOAOMT7: Caffeoyl coenzyme A dependent O-methyltransferase 7; CYP: Cytochrome P450 superfamily of monooxygenases; F6'H1: Feruloyl-CoA 6'-hydroxylase 1; F6'H2: FeruloylCoA 6'-hydroxylase 2; GC/MS: Gas Chromatography/Mass Spectrometry; HCT: Shikimate O-hydroxycinnamoyltransferase; HPLC: High-performance liquid chromatography; LOD: Logarithm of odds; MS: Murashige and Skoog medium; MQM: Multiple QTL mapping; MYB: Superfamily of transcription factors; NASC: Nottingham Arabidopsis stock centre; OMT1: Caffeate O-methyltransferase 1; TSM1: Tapetum-specific O- methyltransferase; PVE: Phenotypic variance explained; SIM: Simple interval mapping; QTL: Quantitative trait loci; WRKY: Superfamily of transcription factors.
\end{abstract}

\section{Competing interests}

The authors declare that they have no competing interests.

\section{Authors' contributions}

JS cultivated the plant material, conducted secondary metabolites isolation, performed the QTL mapping and contributed to the in silico analyses, statistical analyses and the results interpretation. LK, RB and BB conducted the coumarins quantification by HPLC. BB and RB contributed to the statistical analyses. AO was involved in the in silico analyses. AGW contributed to the statistical analyses. EL contributed to the results interpretation. Al received grant support for the project, wrote the paper, design the experiments, interpreted the results, performed the in silico and statistical analyses, and participate in optimization of plant growth and secondary metabolites isolation. All authors read and approved the final manuscript.

\section{Acknowledgements}

This research was supported by the National Science Centre (6815/B/P01/ 2011/40), the Foundation for Polish Science (HOMING Programme) and the LiSMIDoS PhD fellowship (UDA-POKL.04.01.01-00-017/1000). Open access publication cost supported from the project MOBI4Health that has received funding from the European Union's Seventh Framework Programme for research, technological development and demonstration under grant agreement no 316094. We thank Maarten Koornneef from the Max Planck Institute for Plant Breeding Research in Cologne for providing all Arabidopsis seeds used in this study and for critical reading of the manuscript.

\section{Author details}

${ }^{1}$ Intercollegiate Faculty of Biotechnology of University of Gdansk and Medical University of Gdansk, ul. Kladki 24, Gdansk 80-822, Poland. ' Université de Lorraine, UMR 1121 Laboratoire Agronomie et Environnement Nancy-Colmar, 2 avenue de la forêt de Haye, Vandœuvre-lès-Nancy 54505, France. ${ }^{3}$ INRA UMR 1121 Laboratoire Agronomie et Environnement Nancy-Colmar, 2 avenue de la forêt de Haye, Vandœuvre-lès-Nancy 54505, France.

Received: 13 May 2014 Accepted: 9 October 2014

Published online: 18 October 2014

\section{References}

1. Fernie AR, Trethewey RN, Krotzky AJ, Willmitzer L: Metabolite profiling: from diagnostics to systems biology. Nat Rev Mol Cell Biol 2004, 5(9):763-769.

2. Kliebenstein DJ, Osbourn A: Making new molecules - evolution of pathways for novel metabolites in plants. Curr Opin Plant Bio/ 2012, 15(4):415-423.

3. D'Auria JC, Gershenzon J: The secondary metabolism of Arabidopsis thaliana: growing like a weed. Curr Opin Plant Biol 2005, 8(3):308-316.

4. Brotman Y, Riewe D, Lisec J, Meyer RC, Willmitzer L, Altmann T: Identification of enzymatic and regulatory genes of plant metabolism through QTL analysis in Arabidopsis. J Plant Physiol 2011, 168(12):1387-1394.

5. Alonso-Blanco C, Aarts MG, Bentsink L, Keurentjes JJ, Reymond M, Vreugdenhil D, Koornneef M: What has natural variation taught us about plant development, physiology, and adaptation? Plant Cell 2009, 21(7):1877-1896.

6. Koornneef M, onso-Blanco C, Vreugdenhil D: Naturally occurring genetic variation in Arabidopsis thaliana. Annu Rev Plant Biol 2004, 55:141-172.

7. Weigel D: Natural variation in Arabidopsis: from molecular genetics to ecological genomics. Plant Physiol 2012, 158(1):2-22.

8. Keurentjes JJ, Fu J, de $\operatorname{Vos} \mathrm{CH}$, Lommen A, Hall RD, Bino RJ, van der Plas LH, Jansen RC, Vreugdenhil D, Koornneef M: The genetics of plant metabolism. Nat Genet 2006, 38(7):842-849.

9. Lisec J, Meyer RC, Steinfath M, Redestig H, Becher M, Witucka-Wall H, Fiehn O, Torjek O, Selbig J, Altmann T, Willmitzer L: Identification of metabolic and biomass QTL in Arabidopsis thaliana in a parallel analysis of RIL and IL populations. Plant J 2008, 53(6):960-972

10. Rowe HC, Hansen BG, Halkier BA, Kliebenstein DJ: Biochemical networks and epistasis shape the Arabidopsis thaliana metabolome. Plant Cell 2008, 20(5):1199-1216.

11. Kliebenstein DJ, Gershenzon J, Mitchell-Olds T: Comparative quantitative trait loci mapping of aliphatic, indolic and benzylic glucosinolate production in Arabidopsis thaliana leaves and seeds. Genetics 2001, 159(1):359-370.

12. Tholl D, Chen F, Petri J, Gershenzon J, Pichersky E: Two sesquiterpene synthases are responsible for the complex mixture of sesquiterpenes emitted from Arabidopsis flowers. Plant J 2005, 42(5):757-771.

13. Bednarek P, Schneider B, Svatos A, Oldham NJ, Hahlbrock K: Structural complexity, differential response to infection, and tissue specificity of indolic and phenylpropanoid secondary metabolism in Arabidopsis roots. Plant Physiol 2005, 138(2):1058-1070.

14. Kai K, Shimizu B, Mizutani M, Watanabe K, Sakata K: Accumulation of coumarins in Arabidopsis thaliana. Phytochemistry 2006, 67(4):379-386.

15. Kai K, Mizutani M, Kawamura N, Yamamoto R, Tamai M, Yamaguchi H, Sakata K, Shimizu B: Scopoletin is biosynthesized via ortho-hydroxylation of feruloyl CoA by a 2-oxoglutarate-dependent dioxygenase in Arabidopsis thaliana. Plant J 2008, 55(6):989-999.

16. Rohde A, Morreel K, Ralph J, Goeminne G, Hostyn V, De RR, Kushnir S, Van DJ, Joseleau JP, Vuylsteke M, Van DG, Van BJ, Messens E, Boerjan W: Molecular phenotyping of the pal1 and pal2 mutants of Arabidopsis thaliana reveals far-reaching consequences on phenylpropanoid, amino acid, and carbohydrate metabolism. Plant Cell 2004, 16(10):2749-2771.

17. Baillieul F, de Ruffray P, Kauffmann S: Molecular cloning and biological activity of alpha-, beta-, and gamma-megaspermin, three elicitins secreted by Phytophthora megasperma $\mathrm{H}_{2}$ O. Plant Physiol 2003, 131(1):155-166.

18. Stern RS: Psoralen and ultraviolet a light therapy for psoriasis. N Engl J Med 2007, 357(7):682-690.

19. Wulff H, Rauer H, During T, Hanselmann C, Ruff K, Wrisch A, Grissmer S, Hansel W: Alkoxypsoralens, novel nonpeptide blockers of Shaker-type K + channels: synthesis and photoreactivity. J Med Chem 1998, 41(23):4542-4549.

20. Karamat F, Olry A, Doerper S, Vialart G, Ullmann P, Werck-Reichhart D, Bourgaud F, Hehn A: CYP98A22, a phenolic ester 3'-hydroxylase specialized in the synthesis of chlorogenic acid, as a new tool for enhancing the furanocoumarin concentration in Ruta graveolens. BMC Plant Biol 2012, 12:152.

21. Bertolucci SK, Pereira AB, Pinto JE, Oliveira AB, Braga FC: Seasonal variation on the contents of coumarin and kaurane-type diterpenes in Mikania laevigata and M. glomerata leaves under different shade levels. Chem Biodivers 2013, 10(2):288-295.

22. Costet L, Fritig B, Kauffmann S: Scopoletin expression in elicitor-treated and tobacco mosaic virus-infected tobacco plants. Physiol Plant 2002, 115(2):228-235

23. Gnonlonfin BG, Gbaguidi F, Gbenou JD, Sanni A, Brimer L: Changes in scopoletin concentration in cassava chips from four varieties during storage. J Sci Food Agric 2011, 91(13):2344-2347. 
24. Matsumoto S, Mizutani M, Sakata K, Shimizu B: Molecular cloning and functional analysis of the ortho-hydroxylases of p-coumaroyl coenzyme $\mathrm{A} /$ feruloyl coenzyme $\mathrm{A}$ involved in formation of umbelliferone and scopoletin in sweet potato, Ipomoea batatas (L.) Lam. Phytochemistry 2012, 74:49-57.

25. Prats E, Galindo JC, Bazzalo ME, Leon A, Macias FA, Rubiales D, Jorrin JV: Antifungal activity of a new phenolic compound from capitulum of a head rot-resistant sunflower genotype. J Chem Ecol 2007, 33(12):2245-2253.

26. Sargent JA, Skoog F: Effects of indoleacetic acid and kinetin on scopoletin-scopolin levels in relation to growth of tobacco tissues in vitro. Plant Physiol 1960, 35(6):934-941.

27. Schmeda-Hirschmann G, Jordan M, Gerth A, Wilken D, Hormazabal E Tapia AA: Secondary metabolite content in Fabiana imbricata plants and in vitro cultures. Z Naturforsch C 2004, 59(1-2):48-54

28. Taguchi G, Fujikawa S, Yazawa T, Kodaira R, Hayashida N, Shimosaka M, Okazaki M: Scopoletin uptake from culture medium and accumulation in the vacuoles after conversion to scopolin in 2,4-D-treated tobacco cells. Plant Sci 2000, 151(2):153-161.

29. Tal B, Robeson DJ: The metabolism of sunflower phytoalexins ayapin and scopoletin: plant-fungus interactions. Plant Physiol 1986, 82(1):167-172.

30. Gnonlonfin GJB, Sanni A, Brimer L: Review Scopoletin - a coumarin phytoalexin with medicinal properties. Crit Rev Plant Sci 2012, 31:47-56.

31. Vogt T: Phenylpropanoid biosynthesis. Mol Plant 2010, 3(1):2-20.

32. Fraser CM, Chapple C: The phenylpropanoid pathway in Arabidopsis. Arabidopsis Book 2011, 9:e0152.

33. Schoch G, Goepfert S, Morant M, Hehn A, Meyer D, Ullmann P, Werck-Reichhart D: CYP98A3 from Arabidopsis thaliana is a 3'-hydroxylase of phenolic esters, a missing link in the phenylpropanoid pathway. $J$ Biol Chem 2001, 276(39):36566-36574.

34. Ehlting J, Buttner D, Wang Q, Douglas CJ, Somssich IE, Kombrink E: Three 4-coumarate:coenzyme A ligases in Arabidopsis thaliana represent two evolutionarily divergent classes in angiosperms. Plant J 1999, 19(1):9-20.

35. Hamberger B, Hahlbrock K: The 4-coumarate:CoA ligase gene family in Arabidopsis thaliana comprises one rare, sinapate-activating and three commonly occurring isoenzymes. Proc Natl Acad Sci U S A 2004, 101(7):2209-2214.

36. Hoffmann L, Maury S, Martz F, Geoffroy P, Legrand M: Purification, cloning and properties of an acyltransferase controlling shikimate and quinate ester intermediates in phenylpropanoid metabolism. J Biol Chem 2003, 278(1):95-103

37. Hoffmann L, Besseau S, Geoffroy P, Ritzenthaler C, Meyer D, Lapierre C, Pollet B, Legrand M: Silencing of hydroxycinnamoyl-coenzyme A shikimate/quinate hydroxycinnamoyltransferase affects phenylpropanoid biosynthesis. Plant Cell 2004, 16(6):1446-1465

38. Kuhnl T, Koch U, Heller W, Wellmann E: Chlorogenic acid biosynthesis: characterization of a light-induced microsomal 5-O-(4-coumaroyl)-D-quinate/ shikimate 3'-hydroxylase from carrot (Daucus carota L.) cell suspension cultures. Arch Biochem Biophys 1987, 258(1):226-232.

39. Goujon T, Sibout R, Pollet B, Maba B, Nussaume L, Bechtold N, Lu F, Ralph J, Mila I, Barriere Y, Lapierre C, Jouanin L: A new Arabidopsis thaliana mutant deficient in the expression of O-methyltransferase impacts lignins and sinapoyl esters. Plant Mol Biol 2003, 51(6):973-989.

40. Wils CR, Brandt W, Manke K, Vogt T: A single amino acid determines position specificity of an Arabidopsis thaliana CCoAOMT-like O-methyltransferase. FEBS Lett 2013, 587(6):683-689.

41. Grienenberger E, Besseau S, Geoffroy P, Debayle D, Heintz D, Lapierre C, Pollet $B$, Heitz T, Legrand M: A BAHD acyltransferase is expressed in the tapetum of Arabidopsis anthers and is involved in the synthesis of hydroxycinnamoyl spermidines. Plant J 2009, 58(2):246-259.

42. Hino F, Okazaki M, Miura Y: Effect of 2,4-dichlorophenoxyacetic Acid on glucosylation of scopoletin to scopolin in tobacco tissue culture. Plant Physio/ 1982, 69(4):810-813.

43. Bourgaud F, Hehn A, Larbat R, Doerper S, Gontier E, Kellner S, Matern U: Biosynthesis of coumarins in plants: a major pathway still to be unravelled for cytochrome P450 enzymes. Phytochem Rev 2006, 5:293-308.

44. Winter D, Vinegar B, Nahal H, Ammar R, Wilson GV, Provart NJ: An "Electronic Fluorescent Pictograph" browser for exploring and analyzing large-scale biological data sets. PLoS One 2007, 2:e718.

45. Fernie AR, Klee $\mathrm{HJ}$ : The use of natural genetic diversity in the understanding of metabolic organization and regulation. Front Plant Sci 2011, 2:59.
46. Lisec J, Steinfath M, Meyer RC, Selbig J, Melchinger AE, Willmitzer L, Altmann T: Identification of heterotic metabolite QTL in Arabidopsis thaliana RIL and IL populations. Plant J 2009, 59(5):777-788.

47. Grillo MA, Li C, Hammond M, Wang L, Schemske DW: Genetic architecture of flowering time differentiation between locally adapted populations of Arabidopsis thaliana. New Phytol 2013, 197(4):1321-1331.

48. Balasubramanian S, Schwartz C, Singh A, Warthmann N, Kim MC, Maloof JN, Loudet O, Trainer GT, Dabi T, Borevitz JO, Chory J, Weigel D: QTL mapping in new Arabidopsis thaliana advanced intercross-recombinant inbred lines. PLoS One 2009, 4(2):e4318.

49. Price AH: Believe it or not, QTLs are accurate! Trends Plant Sci 2006 11(5):213-216

50. Wayne ML, Mclntyre LM: Combining mapping and arraying: An approach to candidate gene identification. Proc Natl Acad Sci U S A 2002, 99(23):14903-14906

51. Werner JD, Borevitz JO, Warthmann N, Trainer GT, Ecker JR, Chory J, Weigel D: Quantitative trait locus mapping and DNA array hybridization identify an FLM deletion as a cause for natural flowering-time variation. Proc Natl Acad Sci U S A 2005, 102(7):2460-2465.

52. Vigani $G$, Morandini $P$, Murgia I: Searching iron sensors in plants by exploring the link among 2'-OG-dependent dioxygenases, the iron deficiency response and metabolic adjustments occurring under iron deficiency. Front Plant Sci 2013, 4:169

53. Fourcroy P, Siso-Terraza P, Sudre D, Saviron M, Reyt G, Gaymard F, Abadia A, Abadia J, varez-Fernandez A, Briat JF: Involvement of the ABCG37 transporter in secretion of scopoletin and derivatives by Arabidopsis roots in response to iron deficiency. New Phytol 2014, 201(1):155-167.

54. Schmid NB, Giehl RF, Doll S, Mock HP, Strehmel N, Scheel D, Kong X, Hider RC, von Wiren N: Feruloyl-CoA 6'-hydroxylase1-dependent coumarins mediate iron acquisition from alkaline substrates in Arabidopsis. Plant Physiol 2014, 164(1):160-172.

55. Micallef SA, Shiaris MP, Colon-Carmona A: Influence of Arabidopsis thaliana accessions on rhizobacterial communities and natural variation in root exudates. J Exp Bot 2009, 60(6):1729-1742

56. Nguyen C, Bouque V, Bourgaud F, Guckert A: Quantification of Daidzein and Furanocoumarin Conjugates of Psoralea cinerea L. (Leguminosae) Phytochem Anal 1997, 8:27-31.

57. Arends $D$, Prins $P$, Jansen RC, Broman KW: R/qtl: high-throughput multiple QTL mapping. Bioinformatics 2010, 26(23):2990-2992.

58. Broman KW, Wu H, Sen S, Churchill GA: R/qtl: QTL mapping in experimental crosses. Bioinformatics 2003, 19(7):889-890.

59. Thompson JD, Higgins DG, Gibson TJ: CLUSTAL W: improving the sensitivity of progressive multiple sequence alignment through sequence weighting, position-specific gap penalties and weight matrix choice. Nucleic Acids Res 1994, 22:4673-4680

\section{doi:10.1186/s12870-014-0280-9}

Cite this article as: Siwinska et al:: Identification of QTLs affecting scopolin and scopoletin biosynthesis in Arabidopsis thaliana. BMC Plant Biology 2014 14:280.

\section{Submit your next manuscript to BioMed Central and take full advantage of:}

- Convenient online submission

- Thorough peer review

- No space constraints or color figure charges

- Immediate publication on acceptance

- Inclusion in PubMed, CAS, Scopus and Google Scholar

- Research which is freely available for redistribution 